\title{
SOLUTION OF THE INVERSE SCATTERING PROBLEM FOR THE THREE-DIMENSIONAL SCHRÖDINGER EQUATION USING A FREDHOLM INTEGRAL EQUATION*
}

\author{
TUNCAY AKTOSUN† AND CORNELIS VAN DER MEE $\ddagger$
}

\begin{abstract}
It is shown that the inverse scattering problem for the three-dimensional Schrödinger equation with a potential having no spherical symmetry can be solved using a Fredholm integral equation. The integral operator studied here is shown to be compact and self-adjoint with its spectrum in $[-1,1]$. The relationship between solutions of this Fredholm equation and of a related RiemannHilbert problem is also clarified, and it is shown that the Fredholm integral equation is uniquely solvable if and only if the Riemann-Hilbert problem is uniquely solvable.
\end{abstract} equation

Key words. inverse scattering, three-dimensional Schrödinger equation, Fredholm integral

AMS(MOS) subject classifications. 81U40, 35P25, 35Q15, 35R30, 47A40

1. Introduction. Consider the Schrödinger equation in three dimensions

$$
\Delta \psi(k, x, \theta)+k^{2} \psi(k, x, \theta)=V(x) \psi(k, x, \theta),
$$

where $\Delta$ is the Laplacian, $k^{2} \in \mathbf{R}$ is energy, $x \in \mathbf{R}^{3}$ is the space coordinate, and $\theta \in S^{2}$ is a unit vector in $\mathbf{R}^{3}$. We assume that the potential $V(x)$ is real and decreases to zero sufficiently fast as $|x| \rightarrow \infty$. However, we do not assume any spherical symmetry on the potential. As $|x| \rightarrow \infty$, the wavefunction $\psi(k, x, \theta)$ satisfies

$$
\psi(k, x, \theta)=e^{i k \theta \cdot x}+\frac{e^{i k|x|}}{|x|} A\left(k, \frac{x}{|x|}, \theta\right)+o\left(\frac{1}{|x|}\right)
$$

where $A\left(k, \theta, \theta^{\prime}\right)$ is the scattering amplitude. The scattering operator $S\left(k, \theta, \theta^{\prime}\right)$ is then defined by

$$
S\left(k, \theta, \theta^{\prime}\right)=\delta\left(\theta-\theta^{\prime}\right)-\frac{k}{2 \pi i} A\left(k, \theta, \theta^{\prime}\right),
$$

where $\delta$ is the Dirac delta distribution on $S^{2}$. In operator notation (1.3) is written as

$$
S(k)=\mathbf{I}-\frac{k}{2 \pi i} A(k),
$$

where the operators are all defined on $L^{2}\left(S^{2}\right)$, the Hilbert space of complex valued square integrable functions on the unit sphere $S^{2}$ in $\mathbf{R}^{3}$ with the usual inner product.

In this article we study the inverse scattering problem, which consists of recovering $V(x)$ when $S(k)$ is known. Since the main source of information about molecular, atomic, and subatomic particles consists of collision experiments, solving the inverse

\footnotetext{
* Received by the editors August 16, 1989; accepted for publication (in revised form) May 1, 1990.

†Department of Mathematics, Southern Methodist University, Dallas, Texas 75275. The work of this author was supported in part by National Science Foundation grant DMS-9096268.

$\ddagger$ Department of Physics and Astronomy, Free University, Amsterdam, the Netherlands. The work of this author was supported in part by National Science Foundation grant DMS-8823102.
} 
scattering problem is equivalent to determining the forces among particles from scattering data.

For one-dimensional and radial Schrödinger equations, the inverse scattering problem is fairly well understood (at least for certain classes of potentials) [1]. In higher dimensions, however, the situation is quite different. The solution methods developed in higher dimensions include the Newton-Marchenko method [2]-[4], the generalized Gel'fand-Levitan method [2]-[5], the $\bar{\partial}$ method [6]-[9], a method that only relies on backward scattering data [10]-[13], a method that uses the Green's function of Faddeev [14]-[16], and the Wiener-Hopf factorization method [17]. There are still many open problems in multidimensional inverse scattering, and the methods developed are still far from being complete. Newton's recent book [18] gives a comprehensive review of the methods and related open problems in three-dimensional inverse scattering prior to 1989.

The main idea behind both the Newton-Marchenko and generalized Gel'fandLevitan methods is to formulate the inverse scattering problem as a Riemann-Hilbert boundary value problem, to transform this Riemann-Hilbert problem into a nonhomogeneous integral equation where the kernel contains the Fourier transform of the scattering data, and to obtain the potential from the solution of the resulting integral equation. In this paper we give the solution of the three-dimensional inverse scattering problem by generalizing a method by Muskhelishvili and Vekua [19], [20] developed to solve Riemann-Hilbert problems with several unknown functions. In the radial case, Newton and Jost used this method to construct potentials from an $n \times n$ scattering matrix for a system of $n$ ordinary differential equations [21]. Here we generalize the Muskhelishvili-Vekua method (and hence the Newton-Jost method) to solve an operator Riemann-Hilbert problem and thus to obtain the solution of the inverse scattering problem for the three-dimensional Schrödinger equation. In this method, the kernel of the key integral equation is an $n \times n$ matrix valued function whereas in our case we deal with an integral equation whose kernel is an operator valued function. In the Newton-Jost method the inverse scattering problem pertains to a system of $n$ ordinary differential equations with an $n \times n$ scattering matrix; however, in this paper, we deal with the inverse scattering problem for a partial differential equation where the kernel of the key integral equation is an operator valued function. Contrary to the three-dimensional Newton-Marchenko and generalized Gel'fand-Levitan inversion methods, we do not use any Fourier transform in our solution of the inverse scattering problem.

The present paper is organized as follows. In $\S 2$ we identify the class of potentials for which all of the results in this paper are valid, and we state the key RiemannHilbert problem which helps to solve the inverse scattering problem for the threedimensional Schrödinger equation. In $\S 3$, using the Riemann-Hilbert problem, we obtain our fundamental Fredholm integral equation (3.10). In $\S 4$, we show that the Fredholm integral operator of (3.10) is compact and self-adjoint and its spectrum is confined to $[-1,1]$. In $\S 5$, we study the relationship between solutions of our Fredholm integral equation and of the Riemann-Hilbert problem and relate the unique solvability of the Fredholm equation to that of the Riemann-Hilbert problem. In $\S 6$, utilizing the solution of the fundamental Fredholm integral equation, we give the solution of the inverse scattering problem for the three-dimensional Schrödinger equation. Finally in $\S 7$, the conclusion is given.

2. Riemann-Hilbert problem. We first identify the class of potentials for which all of the results in this paper are valid. Except for the third condition given in 
the following definition, these conditions are standard assumptions on the potential [18]. These conditions were instrumental in proving the Hölder continuity of the scattering operator and the existence of a Wiener-Hopf factorization of the scattering operator [17].

DEFINITION 2.1. A potential $V(x)$ is said to belong to the Newton class if $V(x)$ is real valued and measurable and satisfies

1. $\exists a, b>0$ such that

$$
\int_{\mathbf{R}^{3}} d x|V(x)|\left(\frac{|x|+|y|+a}{|x-y|}\right)^{2} \leq b, \quad \forall y \in \mathbf{R}^{3},
$$

2. $\exists c>0, s>\frac{1}{2}$ such that

$$
|V(x)| \leq \frac{c}{\left(1+|x|^{2}\right)^{s}}, \quad \forall x \in \mathbf{R}^{3},
$$

3. $\exists \alpha>0$ and some $\beta \in(0,1]$ such that

$$
\int_{\mathbf{R}^{3}} d x|x|^{\beta}|V(x)| \leq \alpha
$$

4. $k=0$ is not an exceptional point [22]. This condition is satisfied if at zero energy there are neither bound states nor half bound states.

In the Schrödinger equation (1.1), $k$ appears as $k^{2}$ and hence $\psi(-k, x, \theta)$ is a solution whenever $\psi(k, x, \theta)$ is. These two solutions are related to each other as [2]

$$
\psi(k, x, \theta)=\int_{S^{2}} d \theta^{\prime} S\left(k,-\theta, \theta^{\prime}\right) \psi\left(-k, x, \theta^{\prime}\right)
$$

Define

$$
f(k, x, \theta)=e^{-i k \theta \cdot x} \psi(k, x, \theta) .
$$

If the potential satisfies (2.1) and if there are no bound states, for fixed $x$ and $\theta$, the function $f(k, x, \theta)$ has an analytic extension in $k$ to the upper half complex plane $\mathbf{C}^{+}$ and $f(k, x, \theta)=1+O\left(\frac{1}{|k|}\right)$ as $|k| \rightarrow \infty$ there [2]. Similarly $f(-k, x, \theta)$ has an analytic extension in $k$ to the lower half complex plane $\mathbf{C}^{-}$. Hence, using (2.4), we obtain the Riemann-Hilbert problem

$$
f(k, x, \theta)=\int_{S^{2}} d \theta^{\prime} e^{-i k \theta \cdot x} S\left(k,-\theta, \theta^{\prime}\right) e^{-i k \theta^{\prime} \cdot x} f\left(-k, x, \theta^{\prime}\right), \quad k \in \mathbf{R} .
$$

Let us define

$$
\begin{gathered}
G\left(k, x, \theta, \theta^{\prime}\right)=e^{-i k \theta \cdot x} S\left(k,-\theta,-\theta^{\prime}\right) e^{i k \theta^{\prime} \cdot x}, \\
X_{ \pm}(k)=f( \pm k, x, \pm \theta)-1,
\end{gathered}
$$

where $f(k, x, \theta)$ is as in (2.5). Then we can write (2.6) in vector form as

$$
X_{+}(k)=G(k) X_{-}(k)+[G(k)-\mathbf{I}] \hat{1}, \quad k \in \mathbf{R},
$$

where $G(k)$ is the operator on $L^{2}\left(S^{2}\right)$ with its kernel given in (2.7), I is the identity operator on this space, and $\hat{1}$ is the function on $L^{2}\left(S^{2}\right)$ defined as $\hat{1}(\theta)=1, \forall \theta \in$ $S^{2}$. Note that, since $x$ enters (2.9) only as a parameter, we have suppressed the $x$ dependence of all the operators and vectors in (2.9).

If there are bound states, the extension of $f(k, x, \theta)$ in $k$ to $\mathbf{C}^{+}$becomes meromorphic with simple poles on the imaginary axis. A pole at $k=i \kappa$ corresponds to 
a bound state of the Hamiltonian with energy $-\kappa^{2}$. It is possible to remove these simple poles from the Riemann-Hilbert problem by a reduction method [4]. Assume there is a bound state corresponding to a pole at $k=i \kappa$. Using a suitable orthogonal projection $\mathbf{B}$, we form the rational function

$$
\Pi(k)=\mathbf{I}-\mathbf{B}+\frac{k+i \kappa}{k-i \kappa} \mathbf{B}
$$

and define the corresponding reduced quantities

$$
\begin{gathered}
G^{\mathrm{red}}(k)=\Pi(k)^{-1} G(k) \Pi(k) \\
X_{+}^{\mathrm{red}}(k)=\Pi(k)^{-1} X_{+}(k)+\left[\Pi(k)^{-1}-\mathbf{I}\right] \hat{1} \\
X_{-}^{\text {red }}(k)=\Pi(k) X_{-}(k)+[\Pi(k)-\mathbf{I}] \hat{1} .
\end{gathered}
$$

As a result, $X_{+}^{\text {red }}(k)$ does not have a pole at $k=i \kappa$ and $X_{-}^{\text {red }}(k)$ does not have a pole at $k=-i \kappa$. If there is more than one bound state, this procedure must be repeated to remove the finitely many poles corresponding to the bound states; the details can be found in [4]. This eventually leads to the reduced Riemann-Hilbert problem

$$
X_{+}^{\text {red }}(k)=G^{\text {red }}(k) X_{-}^{\text {red }}(k)+\left[G^{\text {red }}(k)-\mathbf{I}\right] \hat{1}, \quad k \in \mathbf{R} .
$$

Once the reduced Riemann-Hilbert problem (2.12) is solved, the solution of the original Riemann-Hilbert problem (2.9) can easily be obtained using (2.10) and (2.11). Hence, in the following sections, without any loss of generality, we will obtain the solution of the Riemann-Hilbert problem assuming that $X_{+}(k)$ and $X_{-}(k)$ have analytic extensions to $\mathbf{C}^{+}$and $\mathbf{C}^{-}$, respectively, and vanish in the norm of $L^{2}\left(S^{2}\right)$ as $k \rightarrow \infty$ from that half plane.

3. Fredholm integral equation. In this section we show that the RiemannHilbert problem posed in (2.9) leads to a Fredholm integral equation, which will be the key equation to solve the inverse scattering problem.

From the Cauchy integral formula we have

$$
\begin{aligned}
& X_{+}(k)=\frac{1}{\pi i} \mathrm{CPV} \int_{-\infty}^{+\infty} d t \frac{X_{+}(t)}{t-k} \\
& X_{-}(k)=-\frac{1}{\pi i} \mathrm{CPV} \int_{-\infty}^{+\infty} d t \frac{X_{-}(t)}{t-k}
\end{aligned}
$$

where CPV denotes the Cauchy principal value. Operating on (3.2) by $G(k)$ and adding the result to (3.1), we obtain

$$
X_{+}(k)+G(k) X_{-}(k)=\frac{1}{\pi i} \mathrm{CPV} \int_{-\infty}^{+\infty} \frac{d t}{t-k}\left[X_{+}(t)-G(k) X_{-}(t)\right] .
$$

Using (2.9) in (3.3) we obtain

$$
\begin{aligned}
2 X_{+}(k) & +[\mathbf{I}-G(k)] \hat{1} \\
= & \frac{1}{\pi i} \operatorname{CPV} \int_{-\infty}^{+\infty} \frac{d t}{t-k}\left(\left[\mathbf{I}-G(k) G(t)^{-1}\right] X_{+}(t)+G(k)\left[\mathbf{I}-G(t)^{-1}\right] \hat{1}\right) .
\end{aligned}
$$

Define the integral operator $K$ whose kernel is given by

$$
K(k, t)=\frac{1}{2 \pi i} \frac{\mathbf{I}-G(k) G(t)^{-1}}{t-k} .
$$


Then we can write (3.4) as

$$
X_{+}(k)-\operatorname{CPV} \int_{-\infty}^{+\infty} d t K(k, t) X_{+}(t)=H(k)
$$

where $H(k)$ is given by

$$
H(k)=\left[G(k)-\mathbf{I}+\int_{-\infty}^{\infty} d t K(k, t)\right] \hat{1} .
$$

If the potential $V(x)$ belongs to the Newton class defined in $\S 2$, the operator $G(k)^{-1}$ is Hölder continuous [17]. Hence the integral in (3.6) is no longer singular and we can drop CPV in front of this integral. Thus, we obtain the regular Fredholm integral equation of the second kind

$$
X_{+}(k)-\int_{-\infty}^{+\infty} d t K(k, t) X_{+}(t)=H(k) .
$$

The Möbius transformation $k \rightarrow \xi=(k-i) /(k+i)$ maps the extended real axis onto the unit circle $\mathbf{T}$, the upper half complex plane $\mathbf{C}^{+}$onto the unit disk $\mathbf{T}^{+}$, and the lower half complex plane $\mathbf{C}^{-}$onto the exterior of the unit disk $\mathbf{T}^{-}$where $\infty$ is considered to be a point of $\mathbf{T}^{-}$. Let $\tilde{S}(\xi)=S(k)$ under this transformation, and let us adopt this notation and use the tilde to denote the Möbius transformed quantity for other functions and operator valued functions throughout the paper.

Let $k \rightarrow \xi=(k-i) /(k+i)$ and $t \rightarrow \eta=(t-i) /(t+i)$ under this Möbius transformation. Defining

$$
Y(\xi)=\frac{\tilde{X}_{+}(\xi)}{1-\xi}=\frac{k+i}{2 i} X_{+}(k)
$$

and

$$
L(\xi)=\frac{\tilde{H}(\xi)}{1-\xi}=\frac{k+i}{2 i} H(k),
$$

we can transform (3.7) into

$$
Y(\xi)-\int_{\mathbf{T}} d \eta \tilde{K}(\xi, \eta) Y(\eta)=L(\xi)
$$

where the kernel of the integral operator $\tilde{K}$ is given by

$$
\tilde{K}(\xi, \eta)=\frac{1}{2 \pi i} \frac{\mathbf{I}-\tilde{G}(\xi) \tilde{G}(\eta)^{-1}}{\eta-\xi} .
$$

Comparing (3.11) with (3.5) we see that $\tilde{K}$ is the Möbius transformed operator for $K$.

4. Properties of the integral operator. In this section we show that the integral operator $\tilde{K}$ in (3.10) is compact and self-adjoint and its spectrum lies in $[-1,1]$.

For $\alpha \in(0,1]$ let $\mathcal{H}_{\alpha}\left(\mathbf{T} ; L^{2}\left(S^{2}\right)\right)$ be the Banach space of Hölder continuous functions $W: \mathbf{T} \rightarrow L^{2}\left(S^{2}\right)$; i.e., the Banach space of all (strongly) continuous functions $W: \mathbf{T} \rightarrow L^{2}\left(S^{2}\right)$ which are bounded with respect to the norm

$$
\|W \mid\|_{\alpha}=\max _{\xi \in \mathbf{T}}\|W(\xi)\|+\sup _{\xi \neq \eta \in \mathbf{T}} \frac{\|W(\xi)-W(\eta)\|}{|\xi-\eta|^{\alpha}} .
$$


Here and in the following $\|\cdot\|$ without any subscript denotes the operator norm on $L^{2}\left(S^{2}\right)$. Let $\mathcal{C}\left(\mathbf{T} ; L^{2}\left(S^{2}\right)\right)$ denote the Banach space of (strongly) continuous functions $W: \mathbf{T} \rightarrow L^{2}\left(S^{2}\right)$ with norm $\|W\|_{\infty}=\max _{\xi \in \mathbf{T}}\|W(\xi)\|$. Finally, for $1 \leq p \leq \infty$ let $L^{p}\left(\mathbf{T} ; L^{2}\left(S^{2}\right)\right)$ denote the Banach space of all strongly measurable functions $W: \mathbf{T} \rightarrow$ $L^{2}\left(S^{2}\right)$ such that $\|W(\cdot)\|$ belongs to $L^{p}(\mathbf{T})[23]$.

Let $\Gamma$ be the singular integral operator on $L^{2}\left(S^{2}\right)$ defined by

$$
(\Gamma f)(\xi)=\frac{1}{\pi i} \operatorname{CPV} \int_{\mathbf{T}} d \eta \frac{f(\eta)}{\eta-\xi} .
$$

Then, from (3.10) and (3.11) it is seen that we can write $\tilde{K}$ as

$$
\tilde{K}=\frac{1}{2}\left(\Gamma-\tilde{G} \Gamma \tilde{G}^{-1}\right),
$$

where $\tilde{G}$ and $\tilde{G}^{-1}$ are operators of multiplication by the respective functions. The space $L^{2}\left(\mathbf{T} ; L^{2}\left(S^{2}\right)\right)$ is a Hilbert space which allows the decomposition into the orthogonal closed subspaces $\mathcal{E}_{+}\left(\mathbf{T} ; L^{2}\left(S^{2}\right)\right)$ and $\mathcal{E}_{-}\left(\mathbf{T} ; L^{2}\left(S^{2}\right)\right)$. Here $\mathcal{E}_{+}\left(\mathbf{T} ; L^{2}\left(S^{2}\right)\right)$ is the subspace of all $L^{2}$-functions which allow for an analytic continuation to $\mathbf{T}^{+}$, while $\mathcal{E}_{-}\left(\mathbf{T} ; L^{2}\left(S^{2}\right)\right)$ is the subspace of $L^{2}$-functions which allow for an analytic continuation to $\mathbf{T}^{-}$that vanishes at infinity. If $f(\xi)$ is an $L^{2}$-function defined on the unit circle $\mathbf{T}$, then using its Fourier series, we have the decomposition

$$
f(\xi)=\sum_{n=-\infty}^{-1} f_{n} \xi^{n}+\sum_{n=0}^{\infty} f_{n} \xi^{n}
$$

as a sum of elements in $\mathcal{E}_{-}\left(\mathbf{T} ; L^{2}\left(S^{2}\right)\right)$ and $\mathcal{E}_{+}\left(\mathbf{T} ; L^{2}\left(S^{2}\right)\right)$, and this decomposition is orthogonal. If we denote the two summations in the above decomposition as $f_{-}$and $f_{+}$, respectively, we obtain

$$
\Gamma f=f_{+}-f_{-} .
$$

Thus, $\Gamma$ is self-adjoint and has unit norm on $L^{2}\left(\mathbf{T} ; L^{2}\left(S^{2}\right)\right)$. More generally, $\Gamma$ is a bounded linear operator on $\mathcal{E}\left(\mathbf{T} ; L^{2}\left(S^{2}\right)\right)$, where $\mathcal{E}$ represents $L^{p}$ with $1<p<\infty$ or $\mathcal{H}_{\gamma}$ with $0<\gamma<1$. This result can be derived from the boundedness of $\Gamma$ on the spaces $\mathcal{E}(\mathbf{T})$ of scalar functions (Theorems I2.1 and I6.1 of [24]) and the density of the linear subspace $\left\{\sum_{j=1}^{n} \varphi_{j}(\cdot) h_{j}: n \in \mathbf{N}, \varphi_{j} \in \mathcal{E}(\mathbf{T}), h_{j} \in L^{2}\left(S^{2}\right)\right\}$ in $\mathcal{E}\left(\mathbf{T} ; L^{2}\left(S^{2}\right)\right)[23]$. sitions.

For potentials identified in Definition 2.1, we will prove the following three propo-

Proposition 4.1. Let $V(x)$ belong to the Newton class. Then the Fredholm integral operator $\tilde{K}$ in $(3.10)$ is compact on $L^{p}\left(\mathbf{T} ; L^{2}\left(S^{2}\right)\right)$ for $1 \leq p \leq \infty$, on $\mathcal{C}\left(\mathbf{T} ; L^{2}\left(S^{2}\right)\right)$, and on $\mathcal{H}_{\gamma}\left(\mathbf{T} ; L^{2}\left(S^{2}\right)\right)$ for $0<\gamma<\mu$. Here $\mu=\beta / 2(1+\beta)$ for $s>1$ in (2.2) and $\mu=\beta(1-s) /\left(\beta-s+\frac{3}{2}\right)$ for $\frac{1}{2}<s<1$ in $(2.2)$, where $\beta \in(0,1]$ is the constant in (2.3) [17].

Proof. Define the integral operator $M$ acting on $L^{2}\left(S^{2}\right)$ as

$$
(M Z)(\xi)=\frac{1}{2 \pi i} \int_{\mathbf{T}} d \eta \frac{\tilde{G}(\eta)-\tilde{G}(\xi)}{\eta-\xi} Z(\eta) .
$$

Then $(\tilde{K} Y)(\xi)=\left(M \tilde{G}^{-1} Y\right)(\xi)$. Due to the fact that the scattering operator $S(k)$ is unitary, the operator $\tilde{G}(\xi)^{-1}$ is bounded with norm $\left\|\tilde{G}^{-1}\right\|=1$. Hence, to prove that $\tilde{K}$ is compact, it is sufficient to prove that $M$ is compact, and we will prove this by showing that $M$ can be approximated by a sequence of finite rank operators. 
It is known [17] that $\tilde{G}(\xi)-I$ is a compact operator on $L^{2}\left(S^{2}\right)$ depending continuously on $\xi \in \mathbf{T}$. Hence it can be approximated by a sequence of finite rank operators $\left\{\tilde{G}_{n}(\xi)-\mathbf{I}\right\}_{n=1}^{\infty}$ given by

$$
\tilde{G}_{n}(\xi)-\mathbf{I}=\sum_{j=-n}^{n} \xi^{j} A_{j}
$$

where $\left\{A_{j}\right\}_{j=-\infty}^{\infty}$ is a sequence of mutually orthogonal finite rank operators on $L^{2}\left(S^{2}\right)$. We then obtain

$$
\frac{\tilde{G}_{n}(\eta)-\tilde{G}_{n}(\xi)}{\eta-\xi}=\sum_{j=1}^{n} \sum_{l=0}^{j-1}\left(\xi^{l} \eta^{j-l-1}-\xi^{l-j} \eta^{-l-1}\right) A_{j}
$$

Using (4.5) in (4.4) we obtain a sequence of operators $\left\{M_{n}\right\}_{n=1}^{\infty}$ given by

$$
\left(M_{n} Y\right)(\xi)=\frac{1}{2 \pi i} \sum_{j=1}^{n}\left(\sum_{l=0}^{j-1} \xi^{l}-\sum_{l=j}^{2 j-1} \xi^{l-2 j}\right) A_{j} \int_{\mathbf{T}} d \eta \eta^{j-l-1} Y(\eta)
$$

Hence, for each $n, M_{n}$ is a finite rank operator. We will complete the proof of our proposition by showing that $M_{n}$ converges to $M$ as $n \rightarrow \infty$ in the function spaces $L^{p}\left(\mathbf{T} ; L^{2}\left(S^{2}\right)\right), \mathcal{C}\left(\mathbf{T} ; L^{2}\left(S^{2}\right)\right)$, and $\mathcal{H}_{\gamma}\left(\mathbf{T} ; L^{2}\left(S^{2}\right)\right)$.

From (4.2) and (4.4), it is seen that $M=\frac{1}{2}(\Gamma \tilde{G}-\tilde{G} \Gamma)$ and $M_{n}=\frac{1}{2}\left(\Gamma \tilde{G}_{n}-\tilde{G}_{n} \Gamma\right)$. Hence, using the boundedness of $\Gamma$ on $\mathcal{E}\left(\mathbf{T} ; L^{2}\left(S^{2}\right)\right)$, where $\mathcal{E}$ is equal to $L^{p}$ with $1<p<\infty$ or equal to $\mathcal{H}_{\gamma}$ with $0<\gamma<\mu$, we have in the operator norm on $\mathcal{E}\left(\mathbf{T} ; L^{2}\left(S^{2}\right)\right)$

$$
\left\|M-M_{n}\right\|_{\mathcal{E}} \leq\|\Gamma\|_{\mathcal{E}}\left\|\tilde{G}-\tilde{G}_{n}\right\|_{\mathcal{E}} \leq c\left\|\tilde{G}-\tilde{G}_{n}\right\|_{\gamma}
$$

for some constant $c>0$. Thus, $M_{n} \rightarrow M$ in the norm of $L^{p}\left(\mathbf{T} ; L^{2}\left(S^{2}\right)\right)$ for $1<p<\infty$ and in the norm of $\mathcal{H}_{\gamma}\left(\mathbf{T} ; L^{2}\left(S^{2}\right)\right)$ for $0<\gamma<\mu$.

It remains to prove the convergence of $M_{n}$ to $M$ as $n \rightarrow \infty$ in the operator norm on $\mathcal{E}\left(\mathbf{T} ; L^{2}\left(S^{2}\right)\right)$. where $\mathcal{E}$ is $L^{1}, L^{\infty}$, or $\mathcal{C}$. First, from (4.4) we obtain

$$
\left\|\left(M-M_{n}\right) Y\right\|_{p} \leq m_{\gamma}\left\|\tilde{G}-\tilde{G}_{n}\right\|\left\|_{\gamma}\right\| Y \|_{p}, \quad p=1, \infty
$$

where

$$
m_{\gamma}=\frac{1}{2 \pi} \int_{\mathbf{T}} \frac{|d \eta|}{|\eta-\xi|^{1-\gamma}}=\frac{1}{2 \pi} \int_{0}^{2 \pi} \frac{d t}{(2-2 \cos t)^{(1-\gamma) / 2}}
$$

is a constant independent of $\xi \in \mathbf{T}$. Then, from (4.7) it follows that $M_{n} \rightarrow M$ in the operator norm on $L^{1}\left(\mathbf{T} ; L^{2}\left(S^{2}\right)\right)$ and on $L^{\infty}\left(\mathbf{T} ; L^{2}\left(S^{2}\right)\right)$. Next, as a result of the dominated convergence theorem for Bochner integrals (Theorem II.3 of [23]), if $\xi \rightarrow \xi_{0}$ in $\mathbf{T}$, then for every $Y \in L^{\infty}\left(\mathbf{T} ; L^{2}\left(S^{2}\right)\right),\left[\left(M-M_{n}\right) Y\right](\xi)$ converges to $\left[\left(M-M_{n}\right) Y\right]\left(\xi_{0}\right)$ in the norm of $L^{2}\left(S^{2}\right)$. Therefore, $M$ and $M_{n} \operatorname{map} L^{\infty}\left(\mathbf{T} ; L^{2}\left(S^{2}\right)\right)$ into $\mathcal{C}\left(\mathbf{T} ; L^{2}\left(S^{2}\right)\right)$. Hence, we obtain from (4.7) that the convergence of $M_{n}$ to $M$ also holds in the operator norm on $\mathcal{C}\left(\mathbf{T} ; L^{2}\left(S^{2}\right)\right)$. Thus, the proof is complete.

If $\lambda$ is a nonzero eigenvalue of $\tilde{K}$, then, by the compactness of $\tilde{K}$ on $\mathcal{E}\left(\mathbf{T} ; L^{2}\left(S^{2}\right)\right)$, there exists some integer $N \geq 1$ such that the kernel of $(\tilde{K}-\lambda \mathbf{I})^{n}$ coincides with the kernel of $(\tilde{K}-\lambda \mathbf{I})^{N}$ if $n \geq N$. The finite dimension of this subspace is called the algebraic multiplicity of $\lambda$. The dimension of the kernel of $\tilde{K}-\lambda \mathbf{I}$ is called the geometric multiplicity of $\lambda$.

As a result of the compactness of $\tilde{K}$, we have the following result. 
COROLLARY 4.2. Let $V(x)$ belong to the Newton class. Then the nonzero eigenvalues of $\tilde{K}$ as well as their (algebraic) multiplicities do not depend on the choice of the function space on which they are defined. As a result, the eigenvectors and generalized eigenvectors of $\tilde{K}$ corresponding to its nonzero eigenvalues belong to each of the spaces $\mathcal{C}\left(\mathbf{T} ; L^{2}\left(S^{2}\right)\right), L^{p}\left(\mathbf{T} ; L^{2}\left(S^{2}\right)\right)$ for $1 \leq p \leq \infty$, and $\mathcal{H}_{\gamma}\left(\mathbf{T} ; L^{2}\left(S^{2}\right)\right)$ for $0<\gamma<\mu$, where $\mu$ is the constant specified in Proposition 4.1.

Proof. Note that for $0<\beta \leq \gamma$ and $1 \leq p \leq q<\infty$ we have

$$
\mathcal{H}_{\gamma} \subset \mathcal{H}_{\beta} \subset \mathcal{C} \subset L^{\infty} \subset L^{q} \subset L^{p}
$$

Consider any of the four pairs of spaces $\left\{L^{\infty}, L^{p}\right\},\left\{L^{q}, L^{p}\right\},\left\{\mathcal{C}, L^{p}\right\}$, and $\left\{\mathcal{H}_{\gamma}, \mathcal{C}\right\}$. In each pair, let $\mathcal{E}_{1}$ denote the first space and $\mathcal{E}_{2}$ denote the second space for functions in $L^{2}\left(S^{2}\right)$. For example, for the first pair, we let $\mathcal{E}_{1}=L^{\infty}\left(\mathbf{T} ; L^{2}\left(S^{2}\right)\right)$ and $\mathcal{E}_{2}=$ $L^{p}\left(\mathbf{T} ; L^{2}\left(S^{2}\right)\right)$. Then for all the pairs, $\mathcal{E}_{1}$ is continuously and densely imbedded in $\mathcal{E}_{2}$. Since $\tilde{K}$ is compact in $\mathcal{E}_{1}$ and in $\mathcal{E}_{2}$, for each nonzero complex number $\lambda$ and natural number $n$, the closure in $\mathcal{E}_{2}$ of the image of $\mathcal{E}_{1}$ under $(\tilde{K}-\lambda \mathbf{I})^{n}$ coincides with the image of $\mathcal{E}_{2}$ under $(\tilde{K}-\lambda \mathbf{I})^{n}$. As a result, the complements of the ranges of $(\tilde{K}-\lambda \mathbf{I})^{n}$ in both $\mathcal{E}_{1}$ and in $\mathcal{E}_{2}$ have the same finite dimension. Since $(\tilde{K}-\lambda \mathbf{I})^{n}$ is a Fredholm operator of index 0 , its kernels in $\mathcal{E}_{1}$ and $\mathcal{E}_{2}$ also have the same finite dimension. As a consequence, the dimensions of the (generalized) eigenspaces for each nonzero eigenvalue of $\tilde{K}$ are the same in $\mathcal{C}\left(\mathbf{T} ; L^{2}\left(S^{2}\right)\right)$, in $L^{p}\left(\mathbf{T} ; L^{2}\left(S^{2}\right)\right)$ for $1 \leq p \leq \infty$, and in $\mathcal{H}_{\gamma}\left(\mathbf{T} ; L^{2}\left(S^{2}\right)\right)$ for $0<\gamma<\mu$, where $\mu$ is the constant specified in Proposition 4.1.

Proposition 4.3. Let the potential $V(x)$ belong to the Newton class. Then $\tilde{K}$ is self-adjoint on $L^{2}\left(\mathbf{T} ; L^{2}\left(S^{2}\right)\right)$. As a result, all eigenvalues of $\tilde{K}$ are real and their algebraic and geometric multiplicities coincide.

Proof. Due to the unitarity of the scattering operator $S(k)$, the operators $\tilde{G}$ and $\tilde{G}^{-1}$ are unitary. Because of (4.3), the singular integral operator $\Gamma$ is self-adjoint. Thus, from (4.2) it follows that $\tilde{K}$ is self-adjoint on $L^{2}\left(\mathbf{T} ; L^{2}\left(S^{2}\right)\right)$.

Proposition 4.4. Let the potential $V(x)$ belong to the Newton class. Then the norm of $\tilde{K}$ in $L^{2}\left(\mathbf{T} ; L^{2}\left(S^{2}\right)\right)$ is bounded above by 1 , and all eigenvalues of $\tilde{K}$ belong to $[-1,1]$.

Proof. From (4.3) it is seen that the singular integral operator $\Gamma$ has unit norm on $L^{2}\left(\mathbf{T} ; L^{2}\left(S^{2}\right)\right)$. Due to their unitarity, the multiplication operators $\tilde{G}$ and $\tilde{G}^{-1}$ each have unit norm. Thus, as seen from (4.2), $\tilde{K}$ has at most unit norm on $L^{2}\left(\mathbf{T} ; L^{2}\left(S^{2}\right)\right)$. Furthermore, by Proposition $4.3, \tilde{K}$ is self-adjoint and hence its spectrum is real. Thus, the spectrum of $\tilde{K}$ lies in $[-1,1]$, and by Corollary 4.2 , the spectrum does not depend on the function space used.

If \pm 1 are not eigenvalues of $\tilde{K}$, the Fredholm integral equation (3.10) has a unique solution which can be obtained by iteration. Since we have shown in Corollary 4.2 that the spectral radius of $\tilde{K}$ does not depend on the function space used, the iteration converges in the norm of any of the spaces mentioned in Proposition 4.1.

5. Relationship between solutions of the Fredholm integral equation and of the Riemann-Hilbert problem. In this section we study the relationship between solutions of the Fredholm integral equation (3.10) and solutions of the Riemann-Hilbert problem on $\mathcal{E}\left(\mathbf{T} ; L^{2}\left(S^{2}\right)\right)$, where $\mathcal{E}$ is either $L^{p}$ with $1<p<\infty$ or $\mathcal{H}_{\gamma}$ with $0<\gamma<\mu, \mu$ being the constant specified in Proposition 4.1. We also investigate the relationship between the partial indices of $\tilde{G}(\xi)[17]$ and the existence and uniqueness of the solution of (3.10). 
Using $F(\xi)=(1 /(1-\xi))[\tilde{G}(\xi)-\mathbf{I}] \hat{1}$, we can transform (2.9) into the RiemannHilbert problem on the unit circle $\mathbf{T}$ to obtain

$$
Y_{+}(\xi)=\tilde{G}(\xi) Y_{-}(\xi)+F(\xi), \quad \xi \in \mathbf{T} .
$$

Let us write (3.10) as

$$
(\mathbf{I}-\tilde{K}) Y=L \text {. }
$$

Note that the nonhomogeneous terms in (5.1) and in (5.2) are related to each other by

$$
L=\frac{1}{2} \tilde{G}(\mathbf{I}+\Gamma) \tilde{G}^{-1} F .
$$

We will relate the solutions of (5.1) with $F \in \mathcal{E}\left(\mathbf{T} ; L^{2}\left(S^{2}\right)\right)$ and $Y_{ \pm} \in \mathcal{E}_{ \pm}\left(\mathbf{T} ; L^{2}\left(S^{2}\right)\right)$ to the solutions of (5.2) with $L, Y \in \mathcal{E}\left(\mathbf{T} ; L^{2}\left(S^{2}\right)\right)$.

As mentioned prior to Proposition 4.1, the singular integral operator $\Gamma$ defined in (4.1) is bounded on $\mathcal{E}\left(\mathbf{T} ; L^{2}\left(S^{2}\right)\right)$. Then $\frac{1}{2}(\mathbf{I}+\Gamma)$ and $\frac{1}{2}(\mathbf{I}-\Gamma)$ are complementary bounded projections onto the subspace $\mathcal{E}_{+}\left(\mathbf{T} ; L^{2}\left(S^{2}\right)\right)$ of all functions in $\mathcal{E}\left(\mathbf{T} ; L^{2}\left(S^{2}\right)\right)$ with an analytic continuation to $\mathbf{T}^{+}$and onto the subspace $\mathcal{E}_{-}\left(\mathbf{T}_{;} L^{2}\left(S^{2}\right)\right)$ of all functions in $\mathcal{E}\left(\mathbf{T} ; L^{2}\left(S^{2}\right)\right)$ with an analytic continuation to $\mathbf{T}^{-}$vanishing at infinity.

THEOREM 5.1. Let $F \in \mathcal{E}\left(\mathbf{T} ; L^{2}\left(S^{2}\right)\right)$. If $Y_{ \pm}$is a solution of $(5.1)$ in $\mathcal{E}_{ \pm}(\mathbf{T}$; $\left.L^{2}\left(S^{2}\right)\right)$, then $Y=Y_{+}$is a solution of (5.2). Conversely, if $Y$ is a solution of (5.2) and $Y \in \mathcal{E}_{+}\left(\mathbf{T} ; L^{2}\left(S^{2}\right)\right)$, then $Y_{ \pm}$, where $Y_{+}=Y$ and $Y_{-}=\tilde{G}^{-1}(Y-F)$, is a solution of (5.1) with $Y_{ \pm} \in \mathcal{E}_{ \pm}\left(\mathbf{T} ; L^{2}\left(S^{2}\right)\right)$.

Proof. Let $Y_{ \pm} \in \mathcal{E}_{ \pm}\left(\mathrm{T} ; L^{2}\left(S^{2}\right)\right)$ be a solution of (5.1). Then using (4.2), (5.3), and $(\mathbf{I} \pm \Gamma) Y_{\mp}=0$, we have

$$
\begin{aligned}
(\mathbf{I}-\tilde{K}) Y_{+} & =\frac{1}{2}(\mathbf{I}-\Gamma) Y_{+}+\frac{1}{2} \tilde{G}(\mathbf{I}+\Gamma) \tilde{G}^{-1} Y_{+} \\
& =\frac{1}{2} \tilde{G}(\mathbf{I}+\Gamma)\left(Y_{-}+\tilde{G}^{-1} F\right)=\frac{1}{2} \tilde{G}(\mathbf{I}+\Gamma) \tilde{G}^{-1} F=L .
\end{aligned}
$$

Conversely, let $Y \in \mathcal{E}_{+}\left(\mathbf{T} ; L^{2}\left(S^{2}\right)\right)$ be a solution of (5.2). Clearly $Y_{+}=Y$ and $Y_{-}=\tilde{G}^{-1}(Y-F)$ satisfy $(5.1)$, provided $Y_{-} \in \mathcal{E}_{-}\left(\mathbf{T} ; L^{2}\left(S^{2}\right)\right)$. This follows from

$$
(\mathbf{I}+\Gamma) Y_{-}=\left(\tilde{G}^{-1}+\Gamma \tilde{G}^{-1}\right) Y-(\mathbf{I}+\Gamma) \tilde{G}^{-1} F=2 \tilde{G}^{-1} L-(\mathbf{I}+\Gamma) \tilde{G}^{-1} F=0,
$$

where we have used (4.2) and (5.3).

From (4.2) and $\Gamma^{2}=\mathbf{I}$ it is immediate that $\tilde{K}^{2}$ and $\Gamma$ commute. Hence, $\tilde{K}^{2}$ maps $\mathcal{E}_{+}\left(\mathbf{T} ; L^{2}\left(S^{2}\right)\right)$ into $\mathcal{E}_{+}\left(\mathbf{T} ; L^{2}\left(S^{2}\right)\right)$ and $\mathcal{E}_{-}\left(\mathbf{T} ; L^{2}\left(S^{2}\right)\right)$ into $\mathcal{E}_{-}\left(\mathbf{T} ; L^{2}\left(S^{2}\right)\right)$. Thus, using the compactness of $\tilde{K}^{2}$, we can decompose the kernel and range of $\mathbf{I}-\tilde{K}^{2}$ as (5.4)

$$
\operatorname{Ran}\left(\mathbf{I}-\tilde{K}^{2}\right)=\left\{\operatorname{Ran}\left(\mathbf{I}-\tilde{K}^{2}\right) \cap \mathcal{E}_{+}\left(\mathbf{T} ; L^{2}\left(S^{2}\right)\right)\right\} \oplus\left\{\operatorname{Ran}\left(\mathbf{I}-\tilde{K}^{2}\right) \cap \mathcal{E}_{-}\left(\mathbf{T} ; L^{2}\left(S^{2}\right)\right)\right\}
$$

and

$$
\operatorname{Ker}\left(\mathbf{I}-\tilde{K}^{2}\right)=\left\{\operatorname{Ker}\left(\mathbf{I}-\tilde{K}^{2}\right) \cap \mathcal{E}_{-}\left(\mathbf{T} ; L^{2}\left(S^{2}\right)\right)\right\} \oplus\left\{\operatorname{Ker}\left(\mathbf{I}-\tilde{K}^{2}\right) \cap \mathcal{E}_{-}\left(\mathbf{T} ; L^{2}\left(S^{2}\right)\right)\right\}
$$

PROPOSITION 5.2. Let $F \in \mathcal{E}\left(\mathbf{T} ; L^{2}\left(S^{2}\right)\right)$. Then there exists at least one solution of (5.2) if and only if there exists at least one solution of the equation

$$
\left(\mathbf{I}-\tilde{K}^{2}\right) Z=(\mathbf{I}+\tilde{K}) L .
$$

Moreover, if it exists, it is possible to choose the solution $Z$ of $(5.5)$ in $\mathcal{E}_{+}\left(\mathbf{T} ; L^{2}\left(S^{2}\right)\right)$, but this solution may not satisfy (5.2).

Proof. If $Y$ is a solution of $(5.2)$ in $\mathcal{E}\left(\mathbf{T} ; L^{2}\left(S^{2}\right)\right)$, then clearly it is also a solution of (5.5). To prove the converse, let us first take $\mathcal{E}=L^{2}$. The solution of (5.5) exists 
provided $(\mathbf{I}+\tilde{K}) L$ is orthogonal to $\operatorname{Ker}\left(\mathbf{I}-\tilde{K}^{2}\right)$. Let $Z_{0}$ be such that $\left(\mathbf{I}-\tilde{K}^{2}\right) Z_{0}=0$. Writing $Z_{0}=Z_{1}+Z_{2}$ where $\tilde{K} Z_{1}=Z_{1}$ and $\tilde{K} Z_{2}=-Z_{2}$, and using the self-adjointness of $\tilde{K}$, we obtain

$$
\left\langle(\mathbf{I}+\tilde{K}) L, Z_{2}\right\rangle=\left\langle L,(\mathbf{I}+\tilde{K}) Z_{2}\right\rangle=0
$$

and

$$
\left\langle(\mathbf{I}+\tilde{K}) L, Z_{1}\right\rangle=\left\langle L,(\mathbf{I}+\tilde{K}) Z_{1}\right\rangle=2\left\langle L, Z_{1}\right\rangle .
$$

Hence, $(\mathbf{I}+\tilde{K}) L$ is orthogonal to $\operatorname{Ker}\left(\mathbf{I}-\tilde{K}^{2}\right)$ if and only if $L$ is orthogonal to $\operatorname{Ker}(\mathbf{I}-$ $\tilde{K})$. Thus, a solution of (5.5) exists if and only if a solution of (5.2) exists.

Furthermore, from (4.2) and (5.3) we obtain

$$
(\mathbf{I}+\tilde{K}) L=\frac{1}{4}(\mathbf{I}+\Gamma) \tilde{G}(\mathbf{I}+\Gamma) \tilde{G}^{-1} F \in \mathcal{E}_{+}\left(\mathbf{T} ; L^{2}\left(S^{2}\right)\right) .
$$

Then, since $N:=\frac{1}{4}(\mathbf{I}+\Gamma) \tilde{G}(\mathbf{I}+\Gamma) \tilde{G}^{-1}$ has a closed range in each $\mathcal{E}\left(\mathbf{T} ; L^{2}\left(S^{2}\right)\right)$, the image of $L^{2}\left(\mathbf{T} ; L^{2}\left(S^{2}\right)\right)$ under $N$ is the closure in $L^{2}\left(\mathbf{T} ; L^{2}\left(S^{2}\right)\right)$ of the image of $\mathcal{E}\left(\mathbf{T} ; L^{2}\left(S^{2}\right)\right)$ under $N$. Similarly, the image of $L^{2}\left(\mathbf{T} ; L^{2}\left(S^{2}\right)\right)$ under $\mathbf{I}+\tilde{K}$ is the closure in $L^{2}\left(\mathbf{T} ; L^{2}\left(S^{2}\right)\right)$ of the image of $\mathcal{E}\left(\mathbf{T} ; L^{2}\left(S^{2}\right)\right)$ under $\mathbf{I}+\tilde{K}$. Hence, if

$$
N\left[L^{2}\left(\mathbf{T} ; L^{2}\left(S^{2}\right)\right)\right] \subset(\mathbf{I}+\tilde{K})\left[L^{2}\left(\mathbf{T} ; L^{2}\left(S^{2}\right)\right)\right]
$$

as proven above, we also have

$$
N\left[\mathcal{E}\left(\mathbf{T} ; L^{2}\left(S^{2}\right)\right)\right] \subset(\mathbf{I}+\tilde{K})\left[\mathcal{E}\left(\mathbf{T} ; L^{2}\left(S^{2}\right)\right)\right]
$$

for $\mathcal{E}=L^{p}$ with $2<p<\infty$ or $\mathcal{E}=\mathcal{H}_{\gamma}$ with $0<\gamma<\mu$. The same conclusion may be drawn if $\mathcal{E}=L^{p}$ with $1<p<2$, but this time we use the fact that $L^{2}\left(\mathbf{T} ; L^{2}\left(S^{2}\right)\right)$ is continuously and densely imbedded in $\mathcal{E}\left(\mathbf{T} ; L^{2}\left(S^{2}\right)\right)$.

Finally, from (5.5) it is seen that a solution of (5.5) exists provided $(\mathbf{I}+\tilde{K}) L \in$ $\operatorname{Ran}\left(\mathbf{I}-\tilde{K}^{2}\right)$. Then using (5.4) and the invertibility of $\left(\mathbf{I}-\tilde{K}^{2}\right)$ on $\operatorname{Ran}\left(\mathbf{I}-\tilde{K}^{2}\right)$, it appears the solution of (5.5) can be chosen in $\operatorname{Ran}\left(\mathbf{I}-\tilde{K}^{2}\right) \cap \mathcal{E}_{+}$.

The number of linearly independent solutions of the homogeneous RiemannHilbert problem

$$
Y_{+}(\xi)=\tilde{G}(\xi) Y_{-}(\xi), \quad \xi \in \mathbf{T}
$$

is the sum of the positive partial indices of $\tilde{G}(\xi)$. Let $\left\{\rho_{j}\right\}$ denote the set of partial indices of $\tilde{G}(\xi)$. These partial indices arise in the Wiener-Hopf factorization of $\tilde{G}(\xi)$ [17].

A special case of Theorem 5.1 with $L=0$ concludes that any solution $Y_{+}$of (5.6) corresponds to a solution of the homogeneous Fredholm integral equation $(\mathbf{I}-\tilde{K}) Y=0$ in $\mathcal{E}_{+}\left(\mathbf{T} ; L^{2}\left(S^{2}\right)\right)$. As a result,

$$
\sum_{\rho_{j}>0} \rho_{j}=\operatorname{dim}\left\{\operatorname{Ker}(\mathbf{I}-\tilde{K}) \cap \mathcal{E}_{+}\left(\mathbf{T} ; L^{2}\left(S^{2}\right)\right)\right\} .
$$

To obtain an expression for the sum of the negative indices, we consider the homogeneous Riemann-Hilbert problem which is adjoint to (5.6)

$$
Z_{+}(\xi)=\tilde{G}(\xi)^{-1} Z_{-}(\xi), \quad \xi \in \mathbf{T}
$$

where $Z_{ \pm} \in \mathcal{E}_{ \pm}\left(\mathbf{T} ; L^{2}\left(S^{2}\right)\right)$. Then the number of linearly independent solutions of (5.8) is the sum of the positive partial indices of $\tilde{G}(\xi)^{-1}$. Due to the unitarity of $\tilde{G}(\xi)$, 
these indices coincide with the negatives of the negative partial indices of $\tilde{G}(\xi)$ [17]. Using (4.2) and (5.8) we have

$$
(\mathbf{I}+\tilde{K}) Z_{-}=\left(\mathbf{I}+\frac{1}{2} \Gamma-\frac{1}{2} \tilde{G} \Gamma \tilde{G}^{-1}\right) Z_{-}=\frac{1}{2}\left(Z_{-}-\tilde{G} \Gamma Z_{+}\right)=0 .
$$

Conversely, if $Z_{-} \in \mathcal{E}_{-}\left(\mathbf{T} ; L^{2}\left(S^{2}\right)\right)$ and $(\mathbf{I}+\tilde{K}) Z_{-}=0$, then $\tilde{G}^{-1} Z_{-}=\tilde{G}^{-1} Z_{-}$ $\tilde{G}^{-1}(\mathbf{I}+\tilde{K}) Z_{-}=\tilde{G}^{-1} Z_{-}-\frac{1}{2} \tilde{G}^{-1}(\mathbf{I}+\Gamma) Z_{-}-\frac{1}{2}(\mathbf{I}-\Gamma) \tilde{G}^{-1} Z_{-}=\frac{1}{2}(\mathbf{I}+\Gamma) \tilde{G}^{-1} Z_{-} \in$ $\mathcal{E}_{+}\left(\mathbf{T} ; L^{2}\left(S^{2}\right)\right)$. As a result, we find the expression

$$
-\sum_{\rho_{j}<0} \rho_{j}=\operatorname{dim}\left\{\operatorname{Ker}(\mathbf{I}+\tilde{K}) \cap \mathcal{E}_{-}\left(\mathbf{T} ; L^{2}\left(S^{2}\right)\right)\right\} .
$$

The norm of $\tilde{K}$ in $L^{2}\left(\mathbf{T} ; L^{2}\left(S^{2}\right)\right)$, i.e., its spectral radius, can be expressed in terms of the gap between certain subspaces [25], [26] For Hilbert spaces the gap between two closed subspaces $\mathcal{M}_{1}$ and $\mathcal{M}_{2}$ equals $\left\|P_{1}-P_{2}\right\|$ where $P_{1}$ and $P_{2}$ are the orthogonal projections onto $\mathcal{M}_{1}$ and $\mathcal{M}_{2}$, respectively. Now notice that $\Gamma_{ \pm}=\frac{1}{2}(I \pm \Gamma)$ are the orthogonal projections of $\mathcal{E}\left(\mathbf{T} ; L^{2}\left(S^{2}\right)\right)$ onto $\mathcal{E}_{ \pm}\left(\mathbf{T} ; L^{2}\left(S^{2}\right)\right)$ and $\Lambda_{ \pm}=\frac{1}{2} \tilde{G}(\mathbf{I} \pm \Gamma) \tilde{G}^{-1}$ are the orthogonal projections of $\mathcal{E}\left(\mathbf{T} ; L^{2}\left(S^{2}\right)\right)$ onto $\tilde{G}\left[\mathcal{E}_{ \pm}\left(\mathbf{T} ; L^{2}\left(S^{2}\right)\right)\right]$. In terms of these projections, from (4.2) we obtain

$$
\tilde{K}=\Gamma_{+}-\Lambda_{+} \quad \text { and } \quad-\tilde{K}=\Gamma_{-}-\Lambda_{-} .
$$

Hence, for $\mathcal{E}=L^{2}$ we have $\|\tilde{K}\|=\operatorname{gap}\left(\mathcal{E}_{ \pm}\left(\mathbf{T} ; L^{2}\left(S^{2}\right)\right), \tilde{G}\left[\mathcal{E}_{ \pm}\left(\mathbf{T} ; L^{2}\left(S^{2}\right)\right)\right]\right)$.

Using the projections $\Gamma_{ \pm}$and $\Lambda_{ \pm}$, we will now derive more convenient expressions for the sums of the positive and negative partial indices of $\tilde{G}(\xi)$ than (5.7) and (5.9). Observe from (5.10) that

$$
\mathbf{I}-\tilde{K}=\Gamma_{-}+\Lambda_{+} \quad \text { and } \quad \mathbf{I}+\tilde{K}=\Gamma_{+}+\Lambda_{-} .
$$

Then we easily find from (5.7)

$$
\sum_{\rho_{j}>0} \rho_{j}=\operatorname{dim}\left\{\mathcal{E}_{+}\left(\mathbf{T} ; L^{2}\left(S^{2}\right)\right) \cap \tilde{G}\left[\mathcal{E}_{-}\left(\mathbf{T} ; L^{2}\left(S^{2}\right)\right)\right]\right\}
$$

while we obtain from $(5.9)$

$$
-\sum_{\rho_{j}<0} \rho_{j}=\operatorname{dim}\left\{\mathcal{E}_{-}\left(\mathbf{T} ; L^{2}\left(S^{2}\right)\right) \cap \tilde{G}\left[\mathcal{E}_{+}\left(\mathbf{T} ; L^{2}\left(S^{2}\right)\right)\right]\right\} .
$$

We can now prove the following.

THEOREM 5.3. The following statements are equivalent:

(1) \pm 1 are not eigenvalues of the Fredholm integral operator $\tilde{K}$ of (5.2).

(2) The Riemann-Hilbert problem (5.1) has a unique solution $Y_{ \pm} \in \mathcal{E}_{ \pm}\left(\mathbf{T} ; L^{2}\left(S^{2}\right)\right)$ for every $F \in \mathcal{E}\left(\mathrm{T} ; L^{2}\left(S^{2}\right)\right)$.

(3) There exists a right canonical Wiener-Hopf factorization

$$
\tilde{G}(\xi)=\tilde{G}_{+}(\xi) \tilde{G}_{-}(\xi), \quad \xi \in \mathbf{T},
$$

of $\tilde{G}(\xi)$ where $\tilde{G}_{ \pm}(\xi)$ and $\tilde{G}_{ \pm}(\xi)^{-1}$ belong to $\mathcal{H}_{\gamma}\left(\mathbf{T} ; \mathcal{L}\left(L^{2}\left(S^{2}\right)\right)\right)$ and have an analytic continuation to $\mathbf{T}^{ \pm}$, where $\gamma$ is the constant specified in Proposition 4.1.

(4) There exists a left canonical Wiener-Hopf factorization

$$
\tilde{G}(\xi)=\tilde{\mathcal{G}}_{-}(\xi) \tilde{\mathcal{G}}_{+}(\xi), \quad \xi \in \mathbf{T},
$$

of $\tilde{G}(\xi)$ where $\tilde{\mathcal{G}}_{ \pm}(\xi)$ and $\tilde{\mathcal{G}}_{ \pm}(\xi)^{-1}$ belong to $\mathcal{H}_{\gamma}\left(\mathbf{T} ; \mathcal{L}\left(L^{2}\left(S^{2}\right)\right)\right.$ and have an analytic continuation to $\mathbf{T}^{ \pm}$. 
(5) The operator function $\tilde{G}(\xi)$, or equivalently the operator function $G(k)$ given in (2.7), has no partial indices [17].

(6) The three-dimensional Jost operator [5] exists and is unique [17].

Proof. The equivalence of (2)-(6), as well as the existence of the left and right Wiener-Hopf factorizations of $\tilde{G}(\xi)$, has been proven in [17]. First, let us show that (1) implies (2). Note that from (5.11) we have

$$
(\mathbf{I}-\tilde{K})\left[\mathcal{E}_{ \pm}\left(\mathbf{T} ; L^{2}\left(S^{2}\right)\right)\right] \subset \tilde{G}\left[\mathcal{E}_{ \pm}\left(\mathbf{T} ; L^{2}\left(S^{2}\right)\right)\right] .
$$

Since $\mathbf{I}-\tilde{K}$ is boundedly invertible in the absence of eigenvalues \pm 1 , it must act as a boundedly invertible operator from $\mathcal{E}_{ \pm}\left(\mathbf{T} ; L^{2}\left(S^{2}\right)\right)$ onto $\tilde{G}\left[\mathcal{E}_{ \pm}\left(\mathbf{T} ; L^{2}\left(S^{2}\right)\right)\right]$. As a result, the unique solution of (5.2) with right-hand side (5.3) belongs to $\mathcal{E}_{+}\left(\mathbf{T} ; L^{2}\left(S^{2}\right)\right)$ for every $F \in \mathcal{E}\left(\mathbf{T} ; L^{2}\left(S^{2}\right)\right)$. Then by Theorem 5.1 , we can conclude that (1) implies (2).

To complete the proof of our theorem, it suffices to prove that (3) and (4) together imply (1). Indeed, let (3) and (4) be true. The canonical Wiener-Hopf factorization of $\tilde{G}(\xi)$ exists only when all the partial indices are zero. Thus, from (5.11) and (5.12) we obtain

$$
\mathcal{E}_{ \pm}\left(\mathbf{T} ; L^{2}\left(S^{2}\right)\right) \cap \tilde{G}\left[\mathcal{E}_{\mp}\left(L^{2}\left(S^{2}\right)\right)\right]=\{0\} .
$$

Hence, if $Y \in \operatorname{Ker}(\mathbf{I}-\tilde{K})$, then using (5.11) one obtains

$$
\Gamma_{-} Y=-\Lambda_{+} Y \in \mathcal{E}_{-}\left(\mathbf{T} ; L^{2}\left(S^{2}\right)\right) \cap \tilde{G}\left[\mathcal{E}_{+}\left(\mathbf{T} ; L^{2}\left(S^{2}\right)\right)\right] .
$$

and hence, by virtue of (5.14),

$$
Y \in \mathcal{E}_{+}\left(\mathbf{T} ; L^{2}\left(S^{2}\right)\right) \cap \tilde{G}\left[\mathcal{E}_{-}\left(\mathbf{T}_{;} L^{2}\left(S^{2}\right)\right)\right]
$$

which proves that $\operatorname{Ker}(\mathbf{I}-\tilde{K})=\{0\}$. Hence \pm 1 are not eigenvalues of $\tilde{K}$, and our proof is complete.

The following corollary gives a sufficient condition on the scattering operator so that the Fredholm integral equation (3.10) is uniquely solvable.

COROLLARY 5.4. The Fredholm integral equation (3.10) and the RiemannHilbert problem (2.9) are uniquely solvable when the scattering operator satisfies $\sup _{k \in \mathbf{R}}\|S(k)-\mathbf{I}\|<1$.

Proof. From (4.2) we have $\tilde{K}=\frac{1}{2}(\mathbf{I}-\tilde{G}) \Gamma-\frac{1}{2} \tilde{G} \Gamma \tilde{G}^{-1}(\mathbf{I}-\tilde{G})$. Since $\Gamma, \tilde{G}$, and $\tilde{G}^{-1}$ have unit norms, we then obtain $\|\tilde{K}\| \leq\|\mathbf{I}-\tilde{G}\|$. However, we have $\|\mathbf{I}-\tilde{G}(\xi)\|=$ $\|\mathbf{I}-G(k)\|=\|S(k)-\mathbf{I}\|$. Thus, if $\sup _{k \in \mathbf{R}}\|S(k)-\mathbf{I}\|<1$, we have $\|\tilde{K}\|<1$, and by Theorem 5.3 both the Riemann-Hilbert problem and the Fredholm integral equation are uniquely solvable.

6. Solution of the inverse problem. Once the Riemann-Hilbert problem posed in (2.9) is solved by solving the Fredholm integral equation (3.10), we obtain $f(k, x, \theta)$ given in (3.2) using (3.8) and (2.8). From the Schrödinger equation (1.1) we then obtain the potential as

$$
V(x)=\frac{(\Delta+2 i k \theta \cdot \nabla) X_{+}(k, x, \theta)}{1+X_{+}(k, x, \theta)}
$$

Note that the right-hand side of this equation contains $\theta$ and $k$ whereas these two variables are absent from the left-hand side. Hence the solution of the Riemann-Hilbert problem will lead to a potential only if the right-hand side of (6.1) is independent of $\theta$ and $k$. Below we show that if the so-called miracle condition [2] occurs and the Riemann-Hilbert problem has a unique solution, then the right-hand side of (6.1) is 
independent of $\theta$ and $k$ and becomes equal to a potential function of $x$. In the absence of bound states the proof has been given in [17], and here we give the proof when the bound states are present.

From (2.10) we have

$$
X_{+}(k)=X_{+}^{\mathrm{red}}(k)+[\Pi(k)-\mathbf{I}] \hat{1}+[\Pi(k)-\mathbf{I}] X_{+}^{\mathrm{red}}(k),
$$

where we have again suppressed the $x$-dependence of $X_{+}(k), X_{+}^{\text {red }}(k)$, and $\Pi(k)$. Defining

$$
\begin{gathered}
\eta(\alpha, x, \theta)=\frac{1}{2 \pi} \int_{-\infty}^{\infty} d k X_{+}(k) e^{-i k \alpha}, \\
\eta^{\mathrm{red}}(\alpha, x, \theta)=\frac{1}{2 \pi} \int_{-\infty}^{\infty} d k X_{+}^{\mathrm{red}}(k) e^{-i k \alpha}, \\
\Omega(\alpha, x, \theta)=\frac{1}{2 \pi} \int_{-\infty}^{\infty} d k[\Pi(k)-\mathbf{I}] e^{-i k \alpha},
\end{gathered}
$$

we can take the Fourier transform of (6.2) to obtain

$$
\eta(\alpha, x, \theta)=\eta^{\mathrm{red}}(\alpha, x, \theta)+\Omega(\alpha, x, \theta) \hat{1}+\int_{-\infty}^{\infty} d \beta \Omega(\alpha-\beta, x, \theta) \eta^{\mathrm{red}}(\beta, x, \theta) .
$$

Note that $X_{+}(k)=O\left(\frac{1}{k}\right)$ as $k \rightarrow \pm \infty$ and is analytic in $\mathbf{C}^{+} ; \Pi(k)-\mathbf{I}=O\left(\frac{1}{k}\right)$ as $k \rightarrow \pm \infty$ and is analytic in $\mathbf{C}^{-}$. As a result, $\eta^{\text {red }}(\alpha, x, \theta)=0$ for $\alpha<0$ and $\Omega(\alpha, x, \theta)=0$ for $\alpha>0$. Thus, we can write (6.4) as

$$
\begin{array}{ll}
\eta(\alpha, x, \theta)=\eta^{\mathrm{red}}(\alpha, x, \theta)+\int_{\alpha}^{\infty} d \beta \Omega(\alpha-\beta, x, \theta) \eta^{\mathrm{red}}(\beta, x, \theta), & \alpha>0, \\
\eta(\alpha, x, \theta)=\Omega(\alpha, x, \theta) \hat{1}+\int_{0}^{\infty} d \beta \Omega(\alpha-\beta, x, \theta) \eta^{\mathrm{red}}(\beta, x, \theta), & \alpha<0 .
\end{array}
$$

In the Newton-Marchenko inversion theory [4], the potential $V(x)$ is obtained from (6.5) and (6.6) as

$$
\begin{aligned}
V(x) & =-2 \theta \cdot \nabla \lim _{\alpha \rightarrow 0^{+}}[\eta(\alpha, x, \theta)-\eta(-\alpha, x, \theta)] \\
& =-2 \theta \cdot \nabla \lim _{\alpha \rightarrow 0^{+}}\left[\eta^{\mathrm{red}}(\alpha, x, \theta)-\Omega(\alpha, x, \theta)\right] \hat{1},
\end{aligned}
$$

provided the right-hand side is independent of $\theta$; this $\theta$-independence is known as the "miracle" identity of Newton [4]. If the miracle occurs and the Riemann-Hilbert problem (2.9) has a unique solution, $\eta(\alpha, x, \theta)$ satisfies the equation

$$
\left[\Delta-2 \frac{\partial}{\partial \alpha} \theta \cdot \nabla-V(x)\right] \eta(\alpha, x, \theta)=0 \text {. }
$$

Then we would like to show that (6.7) and (6.8) imply (6.1). To see this, note that from (6.3) we have

$$
i k X_{+}(k)=\lim _{\alpha \rightarrow 0^{+}}[\eta(-\alpha, x, \theta)-\eta(\alpha, x, \theta)]-\int_{-\infty}^{\infty} d \alpha e^{i k \alpha} \frac{\partial \eta(\alpha, x, \theta)}{\partial \alpha} .
$$

Thus, using (6.7) and (6.8) we obtain

$$
[\Delta+2 i k \theta \cdot \nabla-V(x)] X_{+}(k)=V(x)+\int_{-\infty}^{\infty} d \alpha e^{i k \alpha}\left[\Delta-2 \frac{\partial}{\partial \alpha} \theta \cdot \nabla-V(x)\right] \eta(\alpha, x, \theta) .
$$


From this last equation, it is seen that (6.1) is implied by (6.7) and (6.8); hence, whenever the miracle condition of Newton is satisfied and the Riemann-Hilbert problem has a unique solution, the potential of the Schrödinger equation is given by (6.1).

Remark that whenever the scattering operator $S(k)$ is known to have a corresponding potential, it is guaranteed that the right-hand side of (6.1) is independent of $k$ and $\theta$. As a consequence, any of the statements in Theorem 5.3 is sufficient to guarantee that the right-hand side of (6.1) is independent of $k$ and $\theta$.

7. Concluding remarks. The results of this article remain true for any real measurable potential $V(x)$ on $\mathbf{R}^{3}$ without real exceptional points that leads to a scattering matrix $S(k)$ such that $S(k)-\mathbf{I}$ is compact for all $k \in \mathbf{R}$ and $\tilde{S}(\xi)=$ $S(i(1+\xi) /(1-\xi))$ is Hölder continuous in $\xi$ on $\mathbf{T}$. In that case we may generalize our results here to potentials on $\mathbf{R}^{n}$ with $n \geq 2$. ments.

Acknowledgments. The authors are indebted to Roger Newton for his com-

\section{REFERENCES}

[1] K. Chadan and P. C. Sabatier, Inverse Problems in Quantum Scattering Theory, Second ed., Springer-Verlag, New York, 1989.

[2] R. G. Newton, Inverse scattering. II. Three dimensions, J. Math. Phys., 21 (1980), pp. $1698-1715 ; 22$ (1981), p. 631; 23 (1982), p. 693.

[3] _ Inverse scattering. III. Three dimensions, continued, J. Math. Phys., 22 (1981), pp. 2191-2200; 23 (1982), p. 693.

[4] _ Inverse scattering. IV. Three dimensions: generalized Marchenko construction with bound states, J. Math. Phys., 23 (1982), pp. 2257-2265.

[5] - The Gel'fand-Levitan method in the inverse scattering problem in quantum mechanics, Scattering Theory in Mathematical Physics, J.A. Lavita and J.-P. Marchand (Eds.), Reidel, Dordrecht, 1974, pp. 193-225.

[6] A. I. Nachman and M. J. Ablowitz, A multidimensional inverse scattering method, Studies in Appl. Math., 71 (1984), pp. 243-250.

[7] R. BEALS AND R. R. COIFMAN, Multidimensional inverse scattering and nonlinear P.D.E.'s, Proc. Sympos. Pure Math., 43 (1985), pp. 45-70.

[8] - The D-bar approach to inverse scattering and nonlinear evolutions, Phys. D, 18 (1986), pp. 242-249.

[9] R. G. Novikov AND G. M. Henkin, Solution of a multidimensional inverse scattering problem on the basis of generalized dispersion relations, Sov. Math. Dokl., 35 (1987), pp. 153-157. Dokl. Akad. Nauk. SSSR, 292 (1987), pp. 814-818. (In Russian.).

[10] R. T. Prosser, Formal solution of inverse scattering problems, J. Math. Phys., 10 (1969), pp. 1819-1822.

[11] - Formal solution of inverse scattering problems. II, J. Math. Phys., 17 (1976), pp. 1775-1779.

[12] - Formal solution of inverse scattering problems. III, J. Math. Phys., 21 (1980), pp. 2648-2653.

[13] - Formal solution of inverse scattering problems. IV, J. Math. Phys., 23 (1982), pp. 2127-2130.

[14] L. D. FADDEEV, Increasing solutions of the Schrödinger equation, Sov. Phys. Dokl. 10 (1965), pp. 1033-1035. Dokl. Akad. Nauk. SSSR, 165 (1965), pp. 514-517. (In Russian.)

[15] Three-dimensional inverse problem in the quantum theory of scattering, J. Sov. Math., 5 (1976), pp. 334-396. Itogi Nauki i Tekhniki, 3 (1974), pp. 93-180. (In Russian.).

[16] R. G. Newton, A Faddeev-Marchenko method for inverse scattering in three dimensions, Inverse Problems, 1 (1985), pp. 371-380. 
[17] T. AKTOSUN AND C. VAN DER MEE, Inverse scattering problem for the three-dimensional Schrödinger equation and Wiener-Hopf factorization of the scattering operator, J. Math. Phys., 31 (1990), pp. 2172-2180.

[18] R. G. Newton, Inverse Schrödinger Scattering in Three Dimensions, Springer-Verlag, New York, 1989.

[19] N. I. MUskhelishvili AND N. P. VekUa, The Riemann boundary problem for several unknown functions and its application to systems of singular integral equations, Trudy Tbilissk. Mat. Inst., 12 (1943), pp. 1-46. (In Russian.)

[20] N. I. Muskhelishvili, Singular Integral Equations, Noordhoff, Groningen, 1953. Nauka, Moscow, 1946. (In Russian.)

[21] R. G. NEwTON AND R. Jost, The construction of potentials from the S-matrix for systems of differential equations, Nuovo Cimento, 1 (1955), pp. 590-622.

[22] R. G. Newton, Noncentral potentials: The generalized Levinson theorem and the structure of the spectrum, J. Math. Phys., 18 (1977), pp. 1348-1357.

[23] J. Diestel And J. J. Uhl, JR., Vector Measures, Math. Surveys 15, Amer. Math. Soc., Providence, 1977.

[24] I. GoHBERG AND N. KRUPNIK, Einführung in die Theorie der eindimensionalen singulären Integraloperatoren, Birkhäuser, Basel, 1979. Stiinca, Kishinev, 1973. (In Russian.)

[25] T. Kato, Perturbation Theory for Linear Operators, Springer-Verlag, 1966.

[26] I. C. Gohberg AND A. S. MARkus, Two theorems on the gap between subspaces of a Banach space, Uspekhi Mat. Nauk., 14 (1959), pp. 135-140. (In Russian.) 